\title{
Examining local-level factors shaping school nutrition policy implementation in Ontario, Canada
}

\author{
Michelle M Vine ${ }^{1, *}$ and Susan J Elliott ${ }^{2}$ \\ ${ }^{1}$ School of Geography \& Earth Sciences, McMaster University, 1280 Main Street West, Hamilton, ON L8S 4K1, \\ Canada: ${ }^{2}$ School of Public Health \& Health Systems, University of Waterloo, Waterloo, ON, Canada
}

Submitted 18 January 2013: Final revision received 13 June 2013: Accepted 25 July 2013: First published online 19 September 2013

\begin{abstract}
Objective: Increasing numbers of overweight and obese youth draw attention to the school as an important setting for targeted nutrition interventions, given that it is where they spend a majority of their waking time. The objective of the present study was to explore local-level factors shaping the implementation of a school nutrition policy.

Design: In-depth, semi-structured interviews were conducted in person or via the telephone (a maximum of $60 \mathrm{~min}$ ). An interview guide was informed by the Analysis Grid for Environments Linked to Obesity (ANGELO) framework, research objectives and literature. Key themes centred on policy implementation, including facilitators and barriers (i.e. resources, capacity), user satisfaction (i.e. students) and communication strategies.

Setting: Secondary schools in Ontario, Canada.

Subjects: Twenty-two participants from local agencies supporting school nutrition programming $(n 8)$ and secondary-school principals, vice principals and teachers ( $n$ 14) from nine schools across three Ontario school boards.

Results: Results are organized according to environments outlined in the ANGELO framework. The cost of healthy food for sale, revenue loss (economic), proximity of schools to off-site food outlets (physical), the restrictive nature of policy, the role of key stakeholders (political), the role of stigma and school culture (sociocultural) act as local-level barriers to policy implementation.

Conclusions: Gaps in policy implementation include the high cost of food for sale and subsequent revenue generation, the close proximity of internal and external food environments, the need for consultation and communication between stakeholders, and strategies to reduce stigma and improve the school nutrition culture.
\end{abstract}

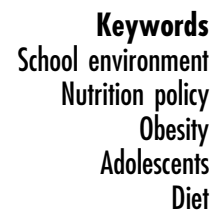

Overweight and obesity are leading public health challenges, particularly among youth, given that an estimated 170 million children $(<18$ years) worldwide are overweight or obese, including $31 \%$ of Canadian youth (2-17 years) ${ }^{(1)}$. Overweight and obese youth are at an increased risk for various chronic diseases and are at greater risk of becoming overweight adults $^{(2)}$. Adiposity has also been linked to bullying behaviours, where overweight and obese youth are more likely than normal-weight youth to be the victims of physical, verbal and relational abuse ${ }^{(3,4)}$.

Eating patterns in childhood and adolescence are important determinants of eating behaviour in adulthood ${ }^{(5)}$. Given that it is where youth spend a large proportion of their waking time, the school is an important setting in which to focus nutrition policies and interventions, particularly at the secondary level where school food environments are less healthier than at the elementary level ${ }^{(6,7)}$. While some progress has been made in improving the school nutrition environment vis-à-vis the development of limiting access to low-nutrient, energy-dense food and beverages ${ }^{(8-10)}$, findings are inconsistent ${ }^{(11,12)}$. Other studies have explored the perceptions and experiences of key school-level stakeholders in the development and implementation of school nutrition policy ${ }^{(13-17)}$. Both of these areas are elaborated on below.

A review of the literature indicates inconsistencies in the health-related outcomes of school nutrition policies. For example, findings of a state-wide school nutrition policy in the USA indicate improvements in the healthfulness of foods consumed by public-school students at lunch ${ }^{(8)}$. A four-state study examining the effectiveness of a multidisciplinary school-based intervention found positive changes in BMI through the consumption of fruits and vegetables and engagement in physical activity ${ }^{(9)}$. In Prince Edward Island, Canada, the findings of Mullally et $a l^{(10)}$ indicate positive changes in student food consumption in accordance with the introduction of a provincial school nutrition policy. However, recent 
findings from Jensen et $a l^{(11)}$ in the USA suggest that no changes in either sweetened beverage consumption or weight status were observed one year after the implementation of a healthy beverage policy. Such findings are consistent with those of Whatley Blum et al. ${ }^{(12)}$ which reveal several barriers to a state-wide nutrition policy, including the availability of other sugar-sweetened beverages, junk food and soda advertisements.

With respect to factors shaping local-level policy implementation, in the USA, findings of a study related to the perceptions of food-service directors and principals of high-school food-service policies suggest the need for stronger communication strategies between school personnel and food-service directors ${ }^{(16)}$. In a large state-wide survey related to school wellness policy implementation in the context of access to healthy food and healthy eating habits, Agron et al. ${ }^{(14)}$ cite a lack of adequate funding, competing priorities/lack of time, a need to garner the support of non-staff stakeholders (e.g. parents, students, community) and inadequate tools and training to support policy development and implementation in schools.

In Canada, the results of Taylor et $a l^{(18)}$ highlight principals' perceptions of key barriers to school nutrition policy implementation in Prince Edward Island, including: lost revenue, the higher cost of healthy foods and the limited availability of policy-compliant foods. In Alberta, evidence suggests that having both a school champion and financial support are integral to the successful adoption of provincial school nutrition guidelines ${ }^{(13)}$. In British Columbia, the results of Rideout et al. ${ }^{(19)}$ indicate that any positive effects of removing snack machines from schools are cancelled by the wide availability of junk food in tuck shops and during school fundraisers.

Inconsistent evidence exists about the success of nutrition policies in improving health outcomes. In Canada, findings indicate that the increased cost of healthy eating combined with the availability of unhealthy food for sale in schools act as barriers to school nutrition policy implementation ${ }^{(13,18-20)}$. The varied landscape of school nutrition policy between provinces, combined with a lack of evidence related to policy components and implementation in Canada, and in secondary schools as opposed to elementary, provides an opportunity to assess local-level factors shaping implementation ${ }^{(20)}$. Given the changing nature of the school nutrition environment in the province of Ontario, Canada, the current research adopted qualitative research methods (i.e. in-depth interviews with key stakeholders) in order to understand how local-level factors shape the implementation of a new provincial-level policy in secondary schools (grades 9-12) in Ontario.

In September 2011, the Ontario Ministry of Education implemented a new School Food and Beverage Policy (PPM 150) ${ }^{(21)}$ across all seventy-two school boards in the province. In conjunction with the Ontario Government's commitment to make schools healthier, PPM 150 strives to contribute to improved educational, attitudinal (e.g. food preferences and eating behaviours) and health-related outcomes, including reducing the risk of students developing chronic diseases such as type 2 diabetes, cancer and heart disease, vis-à-vis nutritional standards (i.e. sell most, sell less, not permitted for sale). PPM 150 allows for ten special-event days where food and beverages sold in schools are exempt from the nutrition standards. Additional requirements include that schools: (i) comply with the Trans Fat Standards set out in Ontario Regulation 200/08 and Regulation 562 related to preparing, serving and storing food and beverages; (ii) develop strategies to reduce the risk of exposure to anaphylactic causative agents; (iii) ensure student access to drinking water during the school day; and (iv) consider the diversity of students and staff through the accommodation of religious and/or cultural needs ${ }^{(21)}$. PPM 150 also recommends that boards, if available and possible, sell Ontarioproduced food and beverages, and avoid offering food and beverages as an incentive to students.

Based on (i) earlier findings ${ }^{(22)}$ which acknowledge a gap in the relationship between regional- and provinciallevel school nutrition policy in the area of food accessibility, nutrition education and vulnerable populations and (ii) inconsistent evidence related to how youth are accessing healthy foods at school in Canadian provinces ${ }^{(20)}$, the objective of the current study was to explore how local-level factors shape policy implementation in Ontario, Canada. In doing so, we examined key stakeholder perceptions of how local-level factors shape the implementation of PPM 150.

The present paper is organized according to the following sections, beginning with an overview of qualitative methods. Results of the research inform a discussion of how local-level factors shape policy implementation, which includes policy implications and directions for future research.

\section{Qualitative methods}

In order to explore the perceptions of key school nutrition stakeholders, the present study adopted a qualitative research design. Overall, key informant interviews ( $n$ 22) were undertaken with community-level participants (from a local public health unit and community agencies with mandates supporting school nutrition; $n$ 8) and school-level participants (i.e. secondary-school principals, vice principals, teachers and administrators from the school board; $n$ 14) from across three boards in Ontario, Canada between December 2011 and March 2012 (see Table 1).

Given the link between neighbourhood characteristics and issues related to food access and dietary patterns ${ }^{(23)}$, school boards were selected from both low- and highincome census tracts (see Table 1). The principal and vice principal of each school received an information letter via email. In participating schools, principals or vice principals either agreed to participate and/or passed the 
Table 1 Sociodemographic comparison of school census tracts (median, participating schools) and province, Ontario, Canada

\begin{tabular}{lrrrr}
\hline & Board 1 & Board 2 & Board 3 & Ontario \\
\hline Average dwelling value (\$CAN) & 204002 & 316943 & 190430 & 297479 \\
Median household income (\$CAN) & 45035 & 80991 & 45902 & 60455 \\
High school education (\%) & $72 \cdot 6$ & $84 \cdot 3$ & $69 \cdot 2$ & $77 \cdot 8$ \\
Immigrant status (\%) & $31 \cdot 7$ & $21 \cdot 7$ & $31 \cdot 4$ & $28 \cdot 3$ \\
Unemployment rate (\%) & $9 \cdot 0$ & $4 \cdot 3$ & $6 \cdot 2$ & $6 \cdot 4$ \\
Low-income households (\%) & $27 \cdot 0$ & $6 \cdot 3$ & $15 \cdot 6$ & $14 \cdot 7$ \\
\hline
\end{tabular}

Adapted from Statistics Canada, 2006 Canadian Census.

Table 2 Interview guide

\begin{tabular}{|c|c|}
\hline Construct & Question \\
\hline \multirow[t]{2}{*}{ Context } & What is your current role? \\
\hline & What brought you to this position? \\
\hline \multirow[t]{2}{*}{ School nutrition in the region } & Tell me about the landscape of school nutrition in your region \\
\hline & $\begin{array}{l}\text { To what extent do you or your organization see nutrition, broadly, and school nutrition, } \\
\text { specifically, as a poverty issue? }\end{array}$ \\
\hline \multirow[t]{4}{*}{ School nutrition policy/programming } & Tell me about the nutrition policy/programmes currently operating in your school \\
\hline & What were/are your school board's expectations for this nutrition policy/programme? \\
\hline & Have these expectations changed since its Inception? \\
\hline & maker and/or programme funder? \\
\hline \multirow[t]{4}{*}{$\begin{array}{l}\text { Perceptions about the policy/ } \\
\text { programme }\end{array}$} & $\begin{array}{l}\text { What is the perception, in your view, about the success of the nutrition policy/programme at the } \\
\text { school level? }\end{array}$ \\
\hline & What are some of the perceived facilitators and barriers to implementation? \\
\hline & Do you think the policy/programme has achieved its expected goals? \\
\hline & $\begin{array}{l}\text { Have there been any targeted evaluation strategies? How do you perceive user satisfaction? } \\
\text { Are there any changes that you think would improve the policy/programme? }\end{array}$ \\
\hline \multirow[t]{2}{*}{ Discussion } & Is there anything else you would like to add that we have not already discussed? \\
\hline & $\begin{array}{l}\text { Is there anyone else you think we should talk to about school nutrition and/or your school's } \\
\text { nutrition policy/programme? }\end{array}$ \\
\hline
\end{tabular}

researcher's contact information on to a family studies/ nutrition teacher for follow-up. Interviews were conducted in person (in a meeting space chosen by the participant) or via telephone by the doctoral student researcher.

An interview guide was informed by previous research findings, the current research objectives and relevant literature (see Table 2). Key topics were related to: school nutrition in the region; perceptions of the school nutrition policy and/or programme in operation in the school, or that which is the focus of organizational activities in community agencies, including facilitators and barriers to implementation; user satisfaction; and strategies for improvement. Interviews were tape-recorded (with written permission) and transcribed verbatim for subsequent thematic analysis. Interviews lasted a maximum of $60 \mathrm{~min}$. The study received clearance from the McMaster University Research Ethics Board (project \#2011 039) and the ethics committees of all three participating school boards.

\section{Data analysis}

The analytic process began during data collection. Data were analysed as they were gathered, helping to shape ongoing data collection (i.e. question refinement, pursuing other avenues of inquiry in depth) ${ }^{(24)}$. The Analysis Grid for Environments Linked to Obesity (ANGELO) framework guided the development of a coding template ${ }^{(25)}$.
The ANGELO framework (see Table 3) is a conceptual model designed to divide the food environment by size (macro and micro) and by type (economic, physical, political, sociocultural). Macro-level sectors (industries or services) influence food intake and micro-level settings (where groups of individuals gather for a specific purpose, usually involving food) are influenced by those sectors. The economic environment refers to the cost of food, while the physical environment is concerned with food availability and accessibility. The political environment helps to determine the rules and guidelines surrounding food. The sociocultural environment is associated with the attitudes and beliefs of individuals as they relate to food. In the current context, the ANGELO framework was utilized to help determine: how locallevel factors shape policy implementation in a school setting; to what extent these factors are considered 'obesogenic'; and how they can be supported and prioritized in future policy interventions ${ }^{(25)}$.

Analyses of a sub-sample ( $n$ 5) of interview transcripts (randomly selected) contributed to the development of the coding template. The four environmental components outlined within the ANGELO framework were mapped onto the data and sub-themes emerged ${ }^{(25)}$. The physical environment included the following sub-themes: education, geography, provision of food to students in school 
Table 3 The Analysis Grid for Environments Linked to Obesity (ANGELO) framework

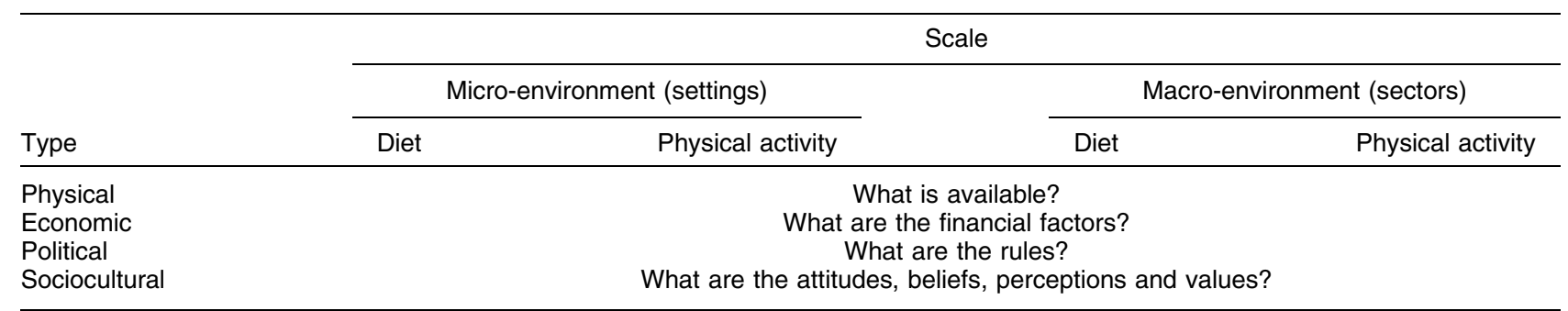

Adapted from Swinburn et al. ${ }^{(25)}$.

venues, nutrition knowledge, school-level capacity and space to store food. Sub-themes within the economic environment included: lack of revenue generation from school cafeteria sales, lack of fundraising capacity, high cost of nutritious food for sale, private-sector competition, lack of student nutrition programme funding and contracts with food providers. The political environment included the following sub-themes: stakeholders, policy guideline compliance, nutrition standards, communication strategies, evaluation and the role of government. The sociocultural environment included sub-themes related to role modelling, school champions, diversity, community partnerships, competing priorities of school, user satisfaction/ dissatisfaction and school culture.

Two researchers undertook an inter-rater reliability exercise, with each independently coding a subset of interviews ( $n$ 3). After reaching 64\% agreement (a sufficient score), this process led to further discussion about meaning and interpretation of codes ${ }^{(26)}$. Data were entered into a qualitative analysis software package (NVivo 8.0) for subsequent thematic analysis, whereby passages of text were selected to support individual codes listed in the coding template. Key themes were generated using a constant comparative method, deductively from the research objectives and inductively as they gradually emerged from the transcripts.

\section{Results}

Given that it was mandatory for Ontario schools to adopt PPM 150 by September 2011, all three school boards were in compliance. However, while board 2 was operating in full compliance under the direction of its board representatives, boards 1 and 3 were operating in compliance, but with more local-level reluctance. This reluctance may at least be partially linked to local-level barriers, as described in the remainder of the paper.

All schools in the study were operating with a full cafeteria, with some also operating separate tuck shops. In seven of nine schools, an outside food-service provider managed cafeteria operations, whereby schools receive a portion of revenue from cafeteria sales. In two schools, instead of having a cafeteria provider, family studies students were responsible for preparing food and operating cafeterias under the supervision of their course instructor. Every school had at least one PPM 150-compliant vending machine, in which they were under contract and for which they receive a portion of revenue of sales. In addition to a cafeteria, one school operated (vis-à-vis students in family studies courses) a restaurant on a for-profit basis, which is accessible to staff and outside customers. All schools were running student nutrition programmes (SNP), offering breakfast, snacks and/or lunch on a regular basis (e.g. daily, two times weekly). SNP were operated by school staff, students and volunteers, and were subsidized by funding from the province, external grants and/or school fundraising initiatives. In some schools, students were asked to pay a small token to access SNP, but payment is not mandatory. Seven of nine schools were within walking distance of off-campus fast-food outlets.

Qualitative results are organized according to the economic, physical, political and sociocultural environments. Within each of these a number of key themes emerged:

1. Economic environment - cost of healthy food for sale, loss of revenue generation.

2. Physical environment - proximity of schools to off-site food outlets, link between healthy eating and student learning.

3. Political environment - restrictive nature of policy guidelines, role of key stakeholders.

4. Sociocultural environment - role of stigma, school culture.

\section{Economic environment}

\section{Cost of healthy food for sale}

Results reveal a link between the new provincial food and beverage policy, the cost of healthy food for sale and a loss of revenue generation. Given the higher cost of policy-compliant (nutritious) food for sale in the cafeteria, respondents recognized that policy guidelines act as a barrier, particularly for vulnerable students, for whom opportunities to access food (healthy or otherwise) may already be constrained at home. For example:

'The consumer is telling us they want certain types of food, and consumers want things that are faster, 
and salad bars are expensive - the simple fact is that you can't eat well at the same price point as you can eat poorly.' (Principal)

While the cost of healthy food for sale was a concern across all schools in the study, it was more pronounced in schools where a larger percentage of the school population was considered low income (i.e. as evidenced by percentage of low-income households in board 1 (see Table 1), in addition to participants' perceptions of the school populations' demographics). Results indicate that stringent nutrition policy guidelines lead some students off campus to fast-food outlets and/or grocery stores, where food is less costly.

\section{Loss of revenue generation}

The focus of policy - selling nutritious foods at school more than $80 \%$ of the time - raises the cost of food for sale and, subsequently, reduces the number of student food purchases at school. As a result, given that schools receive a portion of funding from cafeteria and related food sales (e.g. tuck shops, vending machines), reduced food sales result in lower revenue. Thus, schools are required to engage in more fundraising activities. Opportunities for food-related school fundraising within PPM 150 are limited as bake sales are restricted to ten days per school year.

Given that cafeteria providers appear unable to provide nutritious food within the nutritional confines of the policy at an appropriate cost, schools risk losing revenue and, ultimately, risk losing their cafeteria. A revenue loss means that cafeteria staff risk losing their jobs, which means that students face the double burden of: (i) being unable to afford the healthy food being offered for sale in the cafeteria (despite the option to bring food from home); and (ii) playing a larger role in food production in the cafeteria, as part of their family studies/food courses. For example:

'We are losing money because of it, which is a huge annoyance because we pay staff here through the cafeteria ... So I have to cut my staff, which means more work for the kids, and more work for me as a teacher to produce the food in the cafeteria.' (Teacher)

Respondents revealed the tension between, on one hand, providing nutritious foods to students and, on the other, the revenues that schools gain as a result of selling unhealthful foods. For example:

'The school gets money for having those vending machines in there [to sell diet pop and vitamin water]. I understand there have been cuts; the school is floundering around looking for ways to make money. I understand that, but I also think let's get a little more creative with it, and stop poisoning those kids' bodies to make money to educate them. It just seems counterproductive to me.' (Teacher)

\section{Pbysical environment}

Proximity of schools to off-site food outlets

Respondents revealed that the close geographical proximity of schools to fast-food outlets acted as an obvious barrier to policy implementation. Students attending schools located near a downtown core were more likely to be in walking distance of inexpensive, unhealthy foods and thereby were more likely to purchase these types of foods. As one respondent revealed:

'It [school food and beverage policy] just changes their habits. It doesn't change their habits in what they are eating. It changes their habits in terms of where they are getting the food.' (Principal)

Loss of cafeteria revenue is at least partially related to unrestricted access to off-campus food outlets, resulting in what school-based respondents perceived as competition between internal (i.e. school cafeterias, vending machines, tuck shops) and external community-based food-service providers. For example:

'I do know that our cafeteria profits have significantly decreased, and the kids in the older grades who have access to cars are driving to places in the community, and kids that don't are walking around the street because there is a pizza joint, a sub joint, and a variety store across the road.' (Vice principal)

\section{Link between bealthy eating and student learning}

While some respondents highlighted the link between healthy eating and improved student learning, they also acknowledged the role of hunger as an acute barrier to student learning. The school food and beverage policy was designed to provide nutrition standards and criteria in schools. Although nutritional health was a key priority of many school-level respondents, issues of poverty and hunger also existed. For example:

'The bottom line is that no child should ever be hungry, If they are hungry, they can't learn, you can't teach them. Their priorities are so different.' (Teacher)

According to one vice principal, ensuring that students are being fed healthy foods vis-à-vis school meal programmes (e.g. subsidized food available to students who qualify) was deliberately connected to student learning:

'To make sure they were having a healthy lunch, we actually moved from giving them a lunchtime [cafeteria] voucher where they were very often getting fries and pop, to giving them an actual lunch whereby the right food groups are represented, in terms of nutrition, fats and fibre. Kids need to be fed if they are going to be awake in class and ready to learn, and we all know there is a huge link between nutrition and student learning.' (Vice principal) 
These, and other, types of strategies to promote healthy eating were common in many schools. Such strategies were consistent with PPM 150 in the contexts of nutritional standards and changing student eating behaviours and food preferences.

\section{Political environment}

\section{Restrictive nature of policy guidelines}

Some respondents revealed that the school food and beverage policy (i.e. nutrition standards, nutrition criteria) is restrictive in nature. In this way, a family studies teacher reported some of the barriers related to accessing policycompliant food, consistent with nutrition standards and criteria, available to sell in cafeterias. While many schools have a food-service provider in the cafeteria, in others, students in family studies/food courses are responsible for cafeteria food production:

'Our suppliers also said there are constraints related to quality control in terms of food choices. Because it is fairly new in Ontario, they don't have suppliers that are providing some of the things they want, or some of the things that they may have may not be as tasty as they could be.' (Teacher)

In addition to issues related to food-service provision, some respondents indicated that the policy guidelines were too restrictive in nature, and that secondary students should be given the option to make food-related choices on their own and in moderation. For example:

'My family studies teachers and I really support the spirit of the population, and we always have. We want to teach kids to eat healthy foods to help prepare them. But we also live in the real world, and if we don't teach moderation they are never going to learn it.' (Teacher)

\section{Role of key stakebolders}

There are a number of key stakeholders who are integral to the success of the school food and beverage policy, including: teachers, vice principals, principals, school healthy action teams, government representatives (both policy makers and funders), parents, students, cafeteriaservice providers, and members of the school council. In response to the question 'Whose responsibility is school nutrition in the community?', one key community stakeholder involved in the facilitation of an SNP across board 1 replied:

'Everyone's. I think there is a role for government, for school boards, and for independent committees. There is a role for parents and students to be helping out with the programme, and then for other community members or non-profit groups. I think it is something that benefits everyone when students are learning well in school, and there needs to be more people involved in helping support that programme.' (Community)

While some respondents felt strongly that responsibility for school nutrition was at the school level, others felt that regardless of what schools are doing to promote healthy eating in schools, primary responsibility needed to lie with parents. For example:

'As a result of this change in policy, the schools have a role to play so they must model healthier choices. I made a report to our Board of Trustees with respect to things that were happening with the ministry change, and first and foremost we communicated clearly to the board chair that we can do everything that we want to, but in the end, the responsibility lies with the parents.' (School board)

A public health representative revealed the role of the health unit in promoting healthy eating in schools:

'The public health units are mandated to work with schools to help promote healthy eating. So public health staff, including public health dietitians, nurses, public health inspectors, activity specialists, we all work very closely with the schools.' (Community)

The extent of the role of public health in school nutrition policy implementation deserves further examination.

\section{Sociocultural environment}

Role of stigma

SNP - partially funded through a provincial government ministry - provide breakfast, lunch and snack programmes on a regular basis (daily, or two or three times weekly, depending on the schools' funding model) to students at no cost. Given that SNP do not provide food for sale, they are not guided by the new school food and beverage policy, but are instead guided by 2008 Nutrition Guidelines $^{(27)}$. Both community- and school-level respondents observed the stigma that students experience as a result of not having enough food and/or not having money to purchase food at school. As one respondent illustrated:

'One of the major barriers is finances. I think there is still some reluctance on the part of some students to come in, they are afraid that people will see that it somehow connects to their home life. It is the stigma - that is what they are afraid of.' (Teacher)

Some schools provide students with cafeteria lunch coupons as a way of reducing stigma. Other strategies include inviting teachers and principals to join students for breakfast at the SNP in order to ensure students that they are universal in nature. As one respondent illustrated:

'If there is stigma around the programme, the easiest way to overcome that is to have a teacher say, "The programme is for everyone, I went there for 
breakfast this morning". But when teachers have never attended the programme, then when a child says "Isn't that just for poor kids?" teachers don't have easy responses to that question.' (Community).

SNP are mandated to be policy compliant (vis-à-vis the 2008 Nutrition Guidelines), but given the high cost of nutritious food and a limited operating budget (SNP receive partial ministry funding, donations, and funds incurred by school fundraising), SNP are at risk of being shut down.

\section{School culture}

The culture of the school nutrition environment appears to be largely dependent on the buy-in of key school-level personnel. A community-level stakeholder working in the area of school nutrition revealed the need for administrative buy-in and school champions to lead nutrition initiatives:

'It seems to be a tiny bit hit or miss. School nutrition seems to be two real kinds of important things in my perspective. You need the buy-in from the principal because nothing goes on in a school without the buy-in from the principal. The second big key to success is having a strong person who kind of leads it and organizes it.' (Community)

Teacher support for SNP is a vital component of their promotion and successful implementation at the school level. Role modelling behaviours help to create a culture in schools where nutrition programmes are seen as universal and acceptable.

\section{Discussion}

Given the rising rates of overweight and obesity in youth, and the fact that youth spend a large proportion of their waking time at school, it is important to examine this setting in the context of nutrition policies and interventions, particularly at the secondary-school level where food environments become less healthy ${ }^{(6,7)}$. While some progress has been made in improving the school nutrition environment, stronger policies supporting healthier meals in schools and limiting access to low-nutrient, energydense food are needed ${ }^{(20,28)}$. In the present study, we conducted in-depth, semi-structured interviews with community- and school-level stakeholders in order to explore local-level factors shaping school nutrition policy implementation. The results lead to a series of implications for policy.

First, given the varied landscape of school nutrition policy in Canada and that a national school meal programme has yet to be developed, all cafeterias are revenue driven. Ontario currently lacks a provincial funding strategy tied to the school food and beverage policy, and the high cost of policy-compliant foods for sale acts as a key barrier at the school level ${ }^{(18)}$. The implications of the high cost of nutritious foods are twofold: (i) students choose to go off campus to purchase food elsewhere; and (ii) schools subsequently generate less revenue from food sales. Consistent with the results of Asanin-Dean and Elliott ${ }^{(29)}$ and Story et al. ${ }^{(30)}$, physical environmental influences interact with individual factors (i.e. socioeconomic status) to impact eating behaviours. As such, some populations are more vulnerable than others, particularly in low-income neighbourhoods. The school is a vital setting for nutrition interventions, including offering subsidized breakfast, snack and lunch programmes via SNP. These findings support the need for a stronger financial commitment from both national and provincial governments to provide subsidized policy-compliant foods in schools through PPM 150 and local-level SNP.

Second, findings related to the proximity of schools to off-site fast-food outlets reveal important policy-related implications. Given that secondary-school students have access to the external food environment, a perception exists that schools are in competition with fast food and other off-site food outlets. This finding is consistent with that of Canadian research by Winson ${ }^{(31)}$, which found that the external food environment acted as a structural barrier to the internal school food environment. This is particularly true of Canadian schools where a nationally or provincially supported school meal programme does not exist. Previous research ${ }^{(32,33)}$ highlights a positive association between neighbourhood deprivation and access to fast-food outlets. Our findings indicate that students attending schools located in low-income neighbourhoods may be even more likely to purchase foods from the external food environment, and therefore may be at an increased risk of obesity and other nutritionrelated chronic diseases ${ }^{(2,34)}$. Analyses of the external neighbourhood food environment surrounding schools are warranted in Ontario, Canada, particularly in light of the implementation of PPM 150.

Third, respondents consistently indicated that a range of key stakeholders could play a larger role in school nutrition policy implementation. The literature consistently indicates the need for enhanced communication strategies between policy makers and those who are responsible for policy implementation ${ }^{(16,35)}$. Our findings reveal that consistent communications (e.g. newsletters, public meetings, student assemblies) between all stakeholders (e.g. government, school-level personnel, students, parents, community groups, public health) throughout policy implementation would enhance the process. Consistent with the findings of MacLellan et al. ${ }^{(36)}$, early consultation with key stakeholders in policy development is needed and is an important predictor of the long-term success of such initiatives. In addition, public health dietitians have an important role in policy development and implementation vis-à-vis communication, engagement and education strategies for stakeholders ${ }^{(15)}$. Results of the present research also point to a need for a process to 
create more alignment and consistency between PPM 150 and SNP, which could be supported by public health dietitians given their expertise and knowledge about nutrition standards and criteria.

Fourth, reducing the role of stigma related to students accessing SNP in schools was a key concern. Our findings suggest that student participation in SNP is compromised because of the potential for it to identify them as low income $^{(37)}$. Role modelling healthy eating behaviours can positively impact the extent to which students experience stigma. While school-based strategies to reduce this stigma (i.e. emphasizing the universal nature of SNP, inviting teachers to attend) have been met with varying degrees of success, it is nevertheless an important priority. School culture was also raised as an important opportunity to promote and support PPM 150. For example, school garden initiatives have been shown to promote healthy nutritional intake, academic engagement and students' sense of connection with their school ${ }^{(38)}$. In addition, given that overweight and obese youth are at an increased risk of being the victims of physical and verbal abuse $^{(3,4)}$, there is an opportunity to explore the social environment and social networks in the context of food and issues of body image and disordered eating ${ }^{(39,40)}$.

Our findings highlight important local-level factors that shape provincial-level policy implementation across three Ontario school boards, including the high cost of food for sale and its implications for revenue generation, the proximity of schools to external fast-food sites, the need for consultation and communication between key school nutrition policy and programme stakeholders, strategies to reduce stigma as it relates to accessing SNP, and the importance of the school culture in promoting and implementing successful school nutrition policies.

While the sample size (fourteen school-level participants, across three school boards) was small, we cannot make generalizations about these findings to all school boards in Ontario ( $n 72$ ); however, we can expect these findings to be transferable to other schools in Ontario.

Future research to explore the perceptions of locallevel factors shaping school nutrition policy implementation and sustainability from the perspective of secondary-school students is needed. For example, how important is healthy eating to you? How do you decide what to eat when you are at school? What factors impact how you decide what to eat at school? These and other related questions will be explored in later stages of our research.

\section{Acknowledgements}

Sources of funding: The authors would like to acknowledge funding from the Canadian Heart Health Surveys Follow-up Study, New Emerging Team Grant (CIHR/OHF Application \#69443) and the Canadian Home Economics Foundation. The Canadian Institutes of Health Research and the Canadian Home Economics Foundation had no role in the design, analysis or writing of this article. Conflicts of interest: The authors declare that they have no competing interests. Authors' contributions: M.M.V. contributed to the design of the study, coordinated the study including ethics applications, recruitment, data collection and data analysis, and wrote the manuscript. S.J.E. contributed to the design of the study and contributed to the manuscript. Acknowledgements: The authors would like to acknowledge the research assistance of Naomi Pullen.

\section{References}

1. World Health Organization (2012) Nutrition-Friendly Schools Initiative (NFSI). Geneva: WHO; available at http://www.who.int/nutrition/topics/nut_school_aged/en/

2. Singh AS, Mulder C, Twisk JWR et al. (2008) Tracking of childhood overweight into adulthood: a systematic review of the literature. Obes Rev 9, 474-488.

3. Farhat T, Iannotti RJ \& Simons-Morton BG (2010) Overweight, obesity, youth, and health-risk behaviors. Am J Prev Med 38, 258-267.

4. Janssen I, Craig WM, Boyce WF et al. (2004) Associations between overweight and obesity with bullying behaviors in school-aged children. Pediatrics 113, 1187-1194.

5. Taylor JP, Evers S \& McKenna M (2005) Determinants of healthy eating in children and youth. Can J Public Health 96, Suppl. 3, S20-S26.

6. Kubik MY, Lytle LA, Farbakhsh K et al. (2009) Food use in middle and high school fundraising: does policy support healthful practice? Results from a survey of Minnesota school principals. J Am Diet Assoc 109, 1215-1219.

7. Finkelstein DM, Hill EL \& Whitaker RC (2008) School food environments and policies in US public schools. Pediatrics 122, e251-e259.

8. Cullen KW, Watson K \& Zakeri I (2008) Improvements in middle school student dietary intake after implementation of a Texas public school nutrition policy. Am J Public Health 98, 111-117.

9. Spiegel SA \& Foulk D (2006) Reducing overweight through a multidisciplinary school-based intervention. Obesity (Silver Spring) 14, 88-96.

10. Mullally ML, Taylor JP, Kuhle S et al. (2010) A provincewide school nutrition policy and food consumption in elementary school children in Prince Edward Island. Can J Public Health 101, 40-43.

11. Jensen CD, Sato AF, McMurty MC et al. (2012) School nutrition policy: an evaluation of the Rhode Island Healthier Beverages Policy in schools. ICAN: Infant, Child, \& Adolescent 4, 276-282.

12. Whatley Blum JE, Beaudoin CM, O'Brien LM et al. (2011) Impact of Maine's statewide nutrition policy on high school food environments. Prev Chronic Dis 8, 1-10.

13. Downs SM, Farmer A, Quintanilha M et al. (2012) From paper to practice: barriers to adopting nutrition guidelines in schools. J Nutr Educ Behav 44, 114-122.

14. Agron P, Berends V, Ellis K et al. (2010) School wellness policies: perceptions, barriers, and needs among school leaders and wellness advocates. J Sch Health 80, 527-535.

15. MacLellan D, Holland A, Taylor J et al. (2010) Implementing school nutrition policy: students and parent perspectives. Can J Diet Pract Res 71, 172-177.

16. McDonnell E, Probart C, Weirich JE et al. (2006) School competitive food policies: perceptions of Pennsylvania public high school foodservice directors and principals. J Am Diet Assoc 106, 271-276. 
17. Roberts SM, Pobocik RS, Deek R et al. (2009) A qualitative study of junior high school principals' and school food service directors' experiences with the Texas school nutrition policy. J Nutr Educ Behav 41, 293-299.

18. Taylor JP, MacLellan D, Caiger JM et al. (2011) Implementing elementary school nutrition policy: principal's perspectives. Can J Diet Pract Res 72, e205-e211.

19. Rideout K, Levy-Milne R, Martin C et al. (2007) Food sales outlets, food availability, and the extent of nutrition policy implementation in schools in British Columbia. Can $J$ Public Health 98, 246-250.

20. McKenna ML (2010) Policy options to support healthy eating in schools. Can J Public Health 101, Suppl. 2, S14-S17.

21. Government of Ontario, Ministry of Education (2010) Policy/Program Memorandum No. 150, School Food and Beverage Policy. Toronto, ON: Government of Ontario; available at http://www.edu.gov.on.ca/extra/eng/ppm/ ppm150.pdf

22. Vine MM \& Elliott SJ (2013) Exploring the school nutrition policy environment in Canada using the ANGELO framework. Health Promot Pract (Epublication ahead of print version).

23. Larson NI, Story MT \& Nelson MC (2009) Neighborhood environments: disparities in access to healthy foods in the US. Am J Prev Med 36, 74-81.

24. Pope C, Ziebland S \& Mays N (2000) Qualitative research in health care: analysing qualitative data. BMJ 320, 114-116.

25. Swinburn B, Egger G \& Raza F (1999) Dissecting obesogenic environments: the development and application of a framework for identifying and prioritizing environmental interventions for obesity. Prev Med 29, 563-570.

26. Barbour RS (2001) Checklists for improving rigour in qualitative research: a case of the tail wagging the dog? BMJ 322, 1115-1117.

27. Government of Ontario, Ministry of Children and Youth Services (2008) Student Nutrition Program, Nutrition Guidelines July 2008. Toronto, ON: Government of Ontario; available at http://www.children.gov.on.ca/htdocs/English/ topics/schoolsnacks/nutrition_guidelines.aspx

28. Story M, Nanney MS \& Schwartz MB (2009) Schools and obesity prevention: creating school environments and policies to promote healthy eating and physical activity. Millbank Q 87, 71-100.

29. Asanin-Dean J \& Elliott SJ (2012) Prioritizing obesity in the city. J Urban Health 89, 196-213.

30. Story M, Kaphingst KM, Robinson-O'Brien R et al. (2008) Creating healthy food and eating environments: policy and environmental approaches. Annu Rev Public Health 29, 253-272.

31. Winson A (2008) School food environments and the obesity issue: content, structural determinants, and agency in Canadian high schools. Agric Hum Values 25, 499-511.

32. Pearce J, Blakey T, Witten K et al. (2007) Neighbourhood deprivation and access to fast-food retailing. Am J Prev Med 32, 375-382.

33. Simon PA, Kwan D, Angelescu A et al. (2008) Proximity of fast food restaurants to schools: do neighborhood income and type of school matter? Prev Med 47, 284-288.

34. Raine KD (2004) Overweight and Obesity in Canada: A Population Health Perspective. Ottawa, ON: Canadian Institute for Health Information; available at https:// secure.cihi.ca/free_products/CPHIOverweightandObesity August2004_e.pdf

35. McCormack Brown K, Henry Akintobi T, Pitt S et al. (2004) California school board members' perceptions of factors influencing school nutrition policy. J Sch Health $\mathbf{7 4}$, $52-58$.

36. MacLellan D, Taylor J \& Freeze C (2009) Developing school nutrition policies: enabling and barrier factors. Can Diet Pract Res 70, 166-171.

37. Bhatia R, Jones P \& Reicker Z (2011) Competitive foods, discrimination, and participation in the National School Lunch Program. Am J Public Health 101, 1380-1386.

38. Ozer EJ (2007) The effects of school gardens on students and schools: conceptualization and considerations for maximizing healthy development. Health Educ Behav 34 , 846-863.

39. Trogdon JG, Nonnemaker J \& Pais J (2008) Peer effects in adolescent overweight. $J$ Health Econ 27, 1388-1399.

40. Hutchinson DM \& Rapee RM (2007) Do friends share similar body image and eating problems? The role of social networks and peer influences in early adolescence. Behav Res Ther 45, 1557-1577. 